\title{
The bevacizumab experience in advanced renal cell carcinoma
}

This article was published in the following Dove Press journal:

OncoTargets and Therapy

14 September 2010

Number of times this article has been viewed

\author{
Lauren C Harshman \\ Sandy Srinivas \\ Division of Oncology, Stanford \\ University School of Medicine, \\ Stanford, California, USA
}

Correspondence: Lauren C Harshman Division of Oncology, 875 Blake Wilbur Dr, \#2229 Stanford, California 94305, USA $\mathrm{Tel}+1650-736-8635$

Fax +1650-736-1640

Email laurenhs@stanford.edu

\begin{abstract}
Bevacizumab in combination with interferon alfa is now approved for treatmentnaïve advanced renal cell carcinoma (RCC) in both the US and Europe. Its objective response rates of $30 \%$ and progression-free survival rates of 9-10 months are comparable to the other approved first-line multityrosine kinase inhibitors, sunitinib and pazopanib. Its advantages include a different toxicity profile and assurance of administration compliance given its intravenous formulation. Enthusiasm for its use is blunted by the increased costs, the potential infusion-related reactions, the associated interferon-related toxicities, and the inconvenience of its nonoral formulation. Further study is warranted to assess its efficacy both as a single agent and in combination with the targeted agents and other immunotherapies. With multiple agents now available for the treatment of advanced RCC, identification of patient and tumor-specific biomarkers to inform our choice of first-line therapy and the proper sequence of subsequent therapies is imperative.
\end{abstract}

Keywords: bevacizumab, renal cell carcinoma, interferon alfa

\section{Introduction}

Historically heralded as a disease resistant to most standard chemotherapies, ${ }^{1}$ the treatment arsenal for advanced renal cell carcinoma (RCC) now boasts a relative plethora of active regimens. The discovery of etiological mutations and aberrations in the von Hippel-Lindau ( $V H L)$ gene in the majority of clear-cell RCCs is responsible for a marked shift from toxic immunotherapy-focused treatments that were limited to selected patients to the more broadly applicable "targeted agents." The growing understanding of the biologic changes responsible for clear-cell RCC and the potential role of upregulated hypoxia-inducible genes led to investigation of agents that target the vascular endothelial growth factor (VEGF) pathway. The efficacy and utility of these agents would quickly be established, and in less than 6 years, the limited treatment armamentarium of 2 main immunotherapeutics in 2004 has blossomed into 6 US Food and Drug Administration (FDA)-approved drugs that directly or indirectly regulate VEGF and other growth factor pathways, with many more under investigation. This review will highlight one of these agents, bevacizumab, a monoclonal antibody to the VEGF-A ligand, and will detail the biology behind the rational use, pharmacology, efficacy, safety, tolerability, and role of bevacizumab in the current treatment of advanced RCC. 


\section{Biologic rationale for bevacizumab's efficacy in RCC}

VHL disease is an autosomal dominant disorder characterized by a germline mutation in the $V H L$ gene. It results in a syndrome characterized by benign and malignant tumors of the central nervous system and viscera. Approximately $25 \%-60 \%$ of patients will develop RCC or cysts generally by the time they are 40 years of age (median age 39 years, range $16-67$ years). ${ }^{2} \mathrm{RCC}$ is a common cause of death in this disorder. In contrast, somatic mutations and aberrations in the $V H L$ gene such as through loss of heterozygosity and gene inactivation through methylation are the likely driving force behind the majority of sporadic clear-cell RCCs. ${ }^{3-7}$ Located on chromosome 3 (3p25-26), VHL is tumor suppressor gene that regulates hypoxia-inducible factors (HIF) ${ }^{8,9}$ In situations of normoxia, the VHL protein complexes with HIF1- $\alpha$ and HIF2- $\alpha$, which acts as a signal for proteosome degradation. This signaling is disrupted in situations of hypoxia or aberrant VHL protein such that HIF is not degraded. Constitutively activated HIF results in enhanced glucose uptake and increased expression of new blood vessels (angiogenesis), growth factors, and mitogens promoting tumor cell growth. ${ }^{9}$ Among those factors upregulated by HIF are the proangiogenesis genes, VEGF and platelet-derived growth factor (PDGF). VEGF stimulates endothelial cell growth and angiogenic processes integral to tumor growth. ${ }^{10,11}$ Inhibition of this upregulation has provided the biologic rationale for the anti-VEGF agents whether directed toward the ligand with antibodies such as bevacizumab or at the receptor level with the tyrosine kinase inhibitors (TKI), such as sunitinib or pazopanib.

\section{Clinical development of bevacizumab and pharmacology}

In 1997, Napoleon Ferrara's group reported on their humanization of the murine anti-VEGF monoclonal antibody A.4.6.1 using site-directed mutagenesis of a human framework. ${ }^{12}$ The humanized antibody achieved $90 \%$ inhibition of bovine capillary endothelial cell proliferation and 90\%-95\% tumor reduction in rhabdomyosarcoma and breast carcinoma cell line tumors in nude mice. This recombinant, humanized, monoclonal antibody (rhuMAb) to VEGF-A would later be known as bevacizumab or more commonly to the public as its proprietary name, Avastin ${ }^{\circledR}$. Bevacizumab binds directly to all VEGF-A isoforms which suppresses activation of its receptors 1 (Flt-1) and 2 (KDR) on the surface of endothelial cells. ${ }^{13}$ This neutralization of the ligand-receptor interaction results in inhibition of endothelial cell proliferation and new blood vessel formation.
Initial pharmacokinetic studies of 491 patients, who received bevacizumab $1-20 \mathrm{mg} / \mathrm{kg}$ every $1-3$ weeks, revealed a half-life of approximately 20 days with a time to steady state of 100 days. ${ }^{13}$ Higher bevacizumab clearance correlated with male gender, higher body weight, and increased tumor burden. Doses as low as $0.3 \mathrm{mg} / \mathrm{kg}$ of bevacizumab are able to neutralize all detectable VEGF. ${ }^{14}$

\section{Efficacy}

An initial phase 1 study of single agent bevacizumab in 25 patients with metastatic treatment refractory solid tumors suggested its potential efficacy in RCC. ${ }^{14}$ The drug was well tolerated at doses of $0.1-10 \mathrm{mg} / \mathrm{kg}$ given every 28 days. Although there were no partial responses (PR) or complete responses, 14 patients experienced clinical benefit in the form of minimal responses ( 2 patients) and disease stabilization (12 patients). Interestingly, 1 of the 2 minimal responders and 5 of the 12 patients with stable disease had RCC. No patient developed antibodies to rhuMAb VEGF. This signal of efficacy and tolerability in RCC patients led to a randomized, 3-arm, singlecenter phase 2 trial of bevacizumab in cytokine-refractory patients. ${ }^{15,16}$ In this trial, the efficacy and safety of low-dose and high-dose bevacizumab $(3 \mathrm{mg} / \mathrm{kg}$ and $10 \mathrm{mg} / \mathrm{kg}$ administered every 2 weeks) was compared with a placebo control in 116 patients. The majority (93\%) of the patients had received prior interleukin-2 (IL-2). The trial was stopped early after an interim analysis showed that the $10 \mathrm{mg} / \mathrm{kg}$ dosage improved time to disease progression compared with the control (4.8 months vs 2.5 months, respectively; $P<0.001)$. The lower dosage did not achieve objective response rate (ORR) or significant improvement in time to progression (TTP) over placebo; median TTP was 3 months. The high-dose bevacizumab arm was well tolerated with $10 \%$ of patients achieving a PR and $59 \%$ (23/39) experiencing disease stabilization for an overall disease control rate of nearly $70 \%$.

Seeking to resist the "eventual tumor escape" observed in their bevacizumab monotherapy study, Yang and colleagues ${ }^{16}$ sought to enhance the antiangiogenic effects by combining low-dose bevacizumab with thalidomide. Known for its antiemetic and teratogenic properties, the biologic modifier thalidomide is also thought to be antiangiogenic both directly by inhibiting basic fibroblast growth factor and VEGF and indirectly by blocking tumor necrosis factor $\alpha$, a cytokine known to be secreted by RCC. ${ }^{17-19}$ Thalidomide had shown some efficacy in phase 2 trials in RCC with PR rates upwards of $20 \%$ (range $0 \%-17 \%$ ) and 3-6 months of disease stabilization rates of $8 \%-64 \%$ in 4 small, phase 2 studies. ${ }^{20-23}$ Also of note was that this phase 2 combination trial 
represented the crossover arm of the placebo patients $(n=22)$ in their original 3-arm trial. These crossover patients were randomized either to bevacizumab alone $(n=10)$ or a combination of low-dose bevacizumab and thalidomide $(\mathrm{n}=12)$. Thalidomide $200 \mathrm{mg}$ orally daily was titrated to a maximum of $800 \mathrm{mg}$ daily. Bevacizumab was given as a loading dose of $4.5 \mathrm{mg} / \mathrm{kg}$ intravenously (IV) followed by treatment doses of $3 \mathrm{mg} / \mathrm{kg}$ every 2 weeks. No ORRs were seen in either group. Median TTP did not differ between the 2 groups (2.4 months vs 3.0 months in the combination arm) or when compared with the 37 patients who had been randomized to the low-dose bevacizumab arm in the first stage of the trial.

Further attempting to improve on the efficacy of bevacizumab, Hainsworth and colleagues ${ }^{24}$ investigated combined VEGF and epidermal growth factor receptor (EGFR) blockade by adding the EGFR-TKI, erlotinib, to bevacizumab. Their rationale included prior evidence that transforming growth factor $\alpha$, a known ligand for EGFR, is commonly elevated in RCC and cell line models demonstrating a correlation between EGFR inhibition and suppression of VEGF expression. ${ }^{25,26}$ In their single-arm, phase 2 trial, all patients were nephrectomized, and $68 \%$ were treatment-naïve. Median progression-free survival (PFS) was 11 months, and $60 \%$ remained alive at 18 months. The ORR was $25 \%$ with an additional $61 \%$ experiencing stable disease at 8 weeks. Bukowski et $\mathrm{al}^{27}$ expounded on this work in a multicenter, placebo-controlled, randomized, phase 2 study that compared bevacizumab plus placebo to bevacizumab plus erlotinib in 150 treatment-naïve metastatic RCC patients. No significant difference in RR (13.7\% vs $14 \%$, respectively) or PFS (8.5 months vs 9.9 months, $P=0.58$ ) was observed. An indirect comparison of the median PFS of 8.5 months in the monotherapy group compared favorably with historical data of patients receiving cytokines in this setting, that of 3.1 months for IL-2 and 4.7 months for interferon $\alpha$ (IFN). ${ }^{28,29}$

Seeking to eliminate multiple different tumor angiogenic mechanisms and to take advantage of the known immunotherapy-sensitive nature of RCC, bevacizumab in combination with IFN has been evaluated and studies in combination with IL-2 are ongoing. Two large randomized controlled trials, CALGB 90206 and the global AVOREN trials, have investigated the efficacy of combination IFN and bevacizumab in the first-line treatment of advanced $\mathrm{RCC}^{30-33}$ (Table 1). In both trials, $10 \mathrm{mg} / \mathrm{kg}$ of bevacizumab was administered IV every 2 weeks with IFN ( 9 million units subcutaneously 3 times per week). The primary end point of both trials was initially overall survival (OS).
Table I Comparison between the 2 bevacizumab randomized controlled phase 3 trials in advanced RCC

\begin{tabular}{|c|c|c|}
\hline & CALGB & AVOREN \\
\hline Sample size & 732 & 649 \\
\hline Median age, $y$ & 61 & 61 \\
\hline Male patients,\% & $73 \%$ & $68 \%$ \\
\hline $\begin{array}{l}\text { Performance status ( } \geq 80 \% \\
\text { by KPS or } \geq I \text { by } E C O G)\end{array}$ & $98 \%$ & $94 \%$ \\
\hline Prior nephrectomy & $85 \%$ & $100 \%$ \\
\hline MSKCC risk & & \\
\hline Good & $26 \%$ & $27 \%$ \\
\hline Intermediate & $64 \%$ & $56 \%$ \\
\hline Poor & $10 \%$ & $9 \%$ \\
\hline Unknown & $0 \%$ & $9 \%$ \\
\hline Placebo-controlled & No & Yes \\
\hline $\begin{array}{l}\text { Maximum weeks of IFN- } \alpha \\
\text { allowed by trial }\end{array}$ & None & 52 \\
\hline Assessment of efficacy & Investigator-assessed & Independent \\
\hline Overall survival & $\begin{array}{l}\text { I8.3 vs } 17.4 \\
\text { (stratified, } \\
P=0.097 \text { ) }\end{array}$ & $\begin{array}{l}23.3 \text { vs } 21.3 \\
\text { (stratified, } \\
P=0.1291 \text { ) }\end{array}$ \\
\hline Progression-free survival, mo & 8.5 vs 5.2 & 10.2 vs 5.4 \\
\hline Response rate & $25.5 \%$ vs $13.1 \%$ & $31 \%$ vs $13 \%$ \\
\hline
\end{tabular}

Abbreviations: RCC, renal cell carcinoma; MSKCC, Memorial Sloan-Kettering Cancer Center; IFN, interferon.

Between 2003 and 2005, 732 patients were enrolled in the CALGB trial..$^{32,34}$ The majority were male $(69 \%)$ with an Eastern Cooperative Oncology Group performance status of $0(62 \%)$, nephrectomized $(85 \%)$, and were of intermediate risk by Motzer criteria (64\%). Of the entire cohort, 350 patients received IFN monotherapy and 366 patients received combination therapy. Median follow up was 46.2 months. The primary end point of OS did not significantly differ between the 2 groups: 18.3 months for bevacizumab plus IFN and 17.4 months for IFN monotherapy (unstratified log-rank, $P=0.097)$. After adjusting for the stratification factors of nephrectomy status and number of adverse prognostic risk factors, the hazard ratio (HR) was $0.86(P=0.069)$. Neither nephrectomy status, Motzer risk score, presence of liver metastasis, age, nor gender impacted survival. Although no crossover to the bevacizumab arm was allowed during the trial, $62 \%$ of monotherapy patients and $54 \%$ of combination therapy patients received subsequent VEGF inhibitors. However, the addition of bevacizumab to IFN elicited a significant 3 month benefit in PFS: 8.5 months compared with 5.2 months in the monotherapy arm $(P<0.0001)$. The ORR was significantly improved with bevacizumab: $25.5 \%$ vs $13.1 \%(P<0.0001)$. Median duration of response was 11.9 months with the combination. Limitations to this study included the lack of a placebo control and an independent review of imaging. 
In terms of tolerability, more patients in the combination arm required dose reductions of IFN: $47 \%$ vs $64 \%$, most commonly due to fatigue. The combination arm had more treatment delays ( $31.6 \%$ vs $61.7 \%$ ) and grade $\geq 3$ toxicity ( $80 \%$ vs $63 \%, P<0.001$ ), but notably, duration of treatment was on average 4 months longer. The increased grade $\geq 3$ toxicities were significant for hypertension, anorexia, fatigue, and proteinuria. The majority of the patients $(56 \%)$ in both arms discontinued for disease progression, and the groups were fairly equal in terms of discontinuing treatment due to toxicity: $24 \%$ in the combination arm and $21 \%$ in the monotherapy arm.

During a similar time period, a global placebo-controlled trial, known as AVOREN, investigated the combination of bevacizumab and IFN. ${ }^{30,31}$ The trial was initially designed with a primary end point of OS, but it was later changed to PFS because of concerns over potential confounders including the emergence of preliminary results of the CALGB trial and new second-line therapies that would become available while the trial was in progress. This amendment was permitted by both the European and US regulatory agencies and allowed a preplanned final analysis of PFS data to be performed before the original primary end point OS data would be mature.

In 2004 and 2005, 649 of 821 screened patients were randomized either to bevacizumab and IFN or to placebo plus IFN. Unlike the CALGB trial where IFN was given until disease progression or intolerability, IFN was given for a maximum of 52 weeks. Bevacizumab/placebo was administered until progression or intolerable toxicity. The majority of patients were male with a $\geq 90 \%$ Karnofsky performance status (70\%) and intermediate risk (56\%). In contrast to the CALGB study, patients were required to have had nephrectomy and to have predominantly $(>50 \%)$ clear-cell histology.

Independently assessed median PFS was significantly improved in the bevacizumab arm at 10.2 months compared with 5.4 months in the control group ( $\mathrm{HR}=0.63 ; 95 \%$ confidence interval [CI], $0.52-0.75, P=0.0001)$. All subgroups experienced a PFS benefit with bevacizumab (sex, age, baseline VEGF level, lung metastases, number of metastatic sites, Memorial Sloan-Kettering Cancer Center [MSKCC] score). Overall, 70\% of patients experienced any tumor shrinkage on bevacizumab/IFN compared with 39\% in the control group. ORR by Response Evaluation Criteria In Solid Tumors was $31 \%$ vs $13 \%$. Time to response was 2.2 months with a median duration of 13.5 months in the bevacizumab-containing arm.
Time to treatment failure was also significantly improved over the control group: 7.7 months vs 4.4 months ( $\mathrm{HR}=0.73$; 95\% CI, $0.62-0.87, P=0.0003)$. The median duration of treatment was 9.7 months compared with 5.1 months in favor of the bevacizumab-containing arm. Median dose intensity of IFN was $91 \%$ in the bevacizumab arm and $96 \%$ in the control group. The most commonly reported adverse events in both arms were fatigue (13\% vs $8 \%)$ and asthenia $(11 \%$ vs $7 \%)$. Both fatigue and asthenia were thought more likely IFN-related toxicities, but they did occur with a slightly higher frequency in the bevacizumab group. The most common grade $\geq 3$ adverse event associated with bevacizumab therapy were proteinuria $(8 \%)$ and hypertension $(6 \%)$. Bevacizumab/placebo was discontinued due to toxicity in $23 \%$ of bevacizumab patients compared with $5 \%$ of the control group; IFN was discontinued in $22 \%$ and $12 \%$, respectively.

Given the significant nearly 5-month PFS benefit at the preplanned final analysis, the trial was unblinded early, and the Data Safety and Monitoring Board recommended that even patients who had not progressed on IFN crossover to receive bevacizumab. Thirteen patients eventually crossed-over to receive bevacizumab. Using intent-to-treat analysis, the final median OS was 23.3 months in the investigational arm compared with 21.3 months in the control group (unstratified $\mathrm{HR}=0.91 ; 95 \% \mathrm{CI}, 0.76-1.10, P=0.34)$. The advantage was more robust under analysis stratified for Motzer risk score and region with a $14 \%$ reduction in chance of death $(\mathrm{HR}=0.86$; $95 \% \mathrm{CI}, 0.72-1.04, P=0.13)$ in patients who had received bevacizumab. The benefit of bevacizumab treatment persisted even with reduced doses of IFN to 3-6 MIU 3 times a week with a median OS of 26 months in those patients.

Although not statistically significant, there did appear to be a trend toward a survival benefit with the addition of bevacizumab/IFN to IFN monotherapy. Postprogression therapy was a likely confounder as $55 \%$ of bevacizumab patients and $63 \%$ of control arm patients received a second-line therapy. More than $35 \%$ of these patients received sunitinib or sorafenib, which became available in Europe in July 2006. Although unplanned, an exploratory analysis revealed a nonstatistically significant improvement in median OS in patients who received posttrial TKI therapy in favor of the bevacizumab arm ( $\mathrm{n}=113 \mathrm{vs} 120$, median OS 38.6 months vs 33.5 months; $\mathrm{HR}=0.8 ; 95 \% \mathrm{CI}, 0.56-1.13$, unstratified).

Delving into the question of the relative contribution of IFN to the combination's efficacy, the AVOREN data were retrospectively interrogated to assess the impact of IFN 
dose reductions on $\mathrm{PFS} .{ }^{35}$ Of the 228 patients who received dose reductions of IFN, $64 \%$ were dose reduced to 6 MIU and $31 \%$ to 3 MIU. Approximately $60 \%$ of therapy was at the reduced dose. Upon comparing the full-dose IFN patients to reduced-dose IFN patients, the PFS benefit seen in the total study population persisted with $\mathrm{HR}=0.63(P=0.0026)$ in the reduced-dose group compared with $\mathrm{HR}=0.69(P=0.0007)$ in the full-dose group. There was a considerable reduction in grade 3/4 IFN-related adverse events from that reported within the 6 weeks before the reduction: $44 \%$ vs $18 \%$. Because all patients received at least 1 full dose of IFN ( 9 MIU), whether low-dose IFN is sufficient to achieve similar tumor responses or PFS as full dose could not be assessed. A prospective trial of bevacizumab plus low-dose IFN is ongoing.

On July 31, 2009, the FDA approved bevacizumab in combination with IFN for the treatment of advanced RCC based on the results of the CALGB and AVOREN trials. The somewhat lower ORR and PFS in the CALGB trial was postulated to be related to the higher percentage of intermediate-risk and poor-risk patients, nonnephrectomized patients, the allowance of predominantly nonclear-cell histologies, and the lack of independent review ${ }^{33}$ (Table 1). Given the increased toxicity of the combination and the impressive phase 2 PFS results with bevacizumab monotherapy in a small randomized study evaluating the addition of erlotinib (median PFS $=8.5$ months), its use as a monotherapy is again under investigation in an ongoing randomized phase 2 trial colloquially known as the BeST (Bevacizumab Sorafenib Tensirolimus) trial. ${ }^{27}$

These trials validated the use of antibody-mediated inhibition of the VEGF ligand as a clinically relevant strategy in RCC. ${ }^{33}$ Although a small survival benefit was seen in both studies, it was not statistically significant and likely disadvantaged by the compounding effects of subsequent similar class therapies and improvements in supportive care since the pre-TKI era. The utility of bevacizumab/IFN likely lies in its ability to achieve response, to prolong PFS, and to offer a different side effect profile than the other currently available first-line agents for good-risk and intermediate-risk patients, sunitinib and pazopanib (Table 2). Indirect

Table 2 Comparative incidences of toxicities among bevacizumab and the multityrosine kinase inhibitors used to treat advanced renal cell carcinoma

\begin{tabular}{|c|c|c|c|c|}
\hline & Bevacizumab $^{13,16,31-33}$ & Sunitinib $^{58,61-66}$ & Sorafenib $^{58,61,65,80-83}$ & Pazopanib $^{84-86}$ \\
\hline Fatigue & $<10 \%-93 \%$ & $54 \%-58 \%$ & $29 \%-37 \%$ & $19 \%-46 \%$ \\
\hline Decline in cardiac & $<1 \%$ & $13 \%-2 \mid \%$ & $<1 \%-5 \%^{\mathrm{a}}$ & $<1 \%$ \\
\hline \multicolumn{5}{|l|}{ ejection fraction } \\
\hline Hypertension & $20 \%-28 \%$ & $30 \%$ & $17 \%-22 \%$ & $40 \%-47 \%$ \\
\hline Acute coronary & $1 \%-3 \%$ & NR & $2.9 \%$ & $5 \%$ \\
\hline \multicolumn{5}{|l|}{ syndrome, chest pain } \\
\hline Arteriothrombolic events & $1 \%-4 \%$ & $1.3 \%$ & $1.7 \%$ & $3 \%$ \\
\hline Venothrombolic events & $3 \%-4 \%$ & $2 \%$ & $<1 \%$ & NR \\
\hline Hemorrhage/bleeding & $5 \%-33 \%$ & $18 \%-30 \%$ & $15 \%$ & $16 \%$ \\
\hline Proteinuria & $18 \%-7 \mid \%$ & Case reports ${ }^{\mathrm{b}}$ & Case reports ${ }^{\mathrm{b}}$ & $9 \%$ \\
\hline Rash & $0 \%-<5 \%$ & $24 \%-27 \%$ & $40 \%-41 \%$ & $8 \%-16 \%$ \\
\hline Hand-foot syndrome & $0 \%-<5 \%$ & $21 \%-29 \%$ & $30 \%-60 \%$ & $6 \%-11 \%$ \\
\hline Diarrhea & $20 \%-21 \%$ & $58 \%-61 \%$ & $43 \%-55 \%$ & $52 \%-63 \%$ \\
\hline Nausea & $<10 \%-58 \%$ & $49 \%-52 \%$ & $19 \%-23 \%$ & $26 \%-42 \%$ \\
\hline Vomiting & $<10 \%$ & $28 \%-32 \%$ & $12 \%-16 \%$ & $20 \%-21 \%$ \\
\hline Mucositis/stomatitis & $0 \%-, 5 \%$ & $30 \%-43 \%$ & $5 \%-17 \%$ & $<10 \%$ \\
\hline \multicolumn{5}{|l|}{ Myelosuppression } \\
\hline Leukopenia & NR & $78 \%$ & $>10 \%$ & $35 \%-37 \%$ \\
\hline Neutropenia & $7 \%-43 \%$ & $72 \%-77 \%$ & $18 \%$ & $27 \%-34 \%$ \\
\hline Anemia & $16 \%$ & $71 \%-79 \%$ & $8 \%-44 \%$ & $<10 \%-26 \%$ \\
\hline Thrombocytopenia & $6 \%-10 \%$ & $65 \%-68 \%$ & $12 \%$ & $26 \%-32 \%$ \\
\hline Transaminitis & $8 \%$ & $46 \%-56 \%$ & $1 \%-10 \%$ & $53 \%-54 \%$ \\
\hline Hyperbilirubinemia & $0 \%-5 \%$ & $12 \%-20 \%$ & $<1 \%$ & $28 \%-36 \%$ \\
\hline Hypothyroidism & $<1 \%$ & $14 \%$ & $<1 \%$ & $4 \%$ \\
\hline Infusion reaction & $<3 \%$ & $\mathrm{n} / \mathrm{a}$ & $\mathrm{n} / \mathrm{a}$ & $\mathrm{n} / \mathrm{a}$ \\
\hline
\end{tabular}

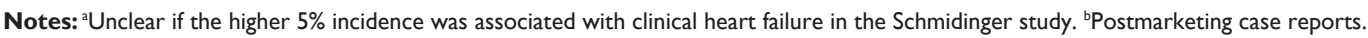

Abbreviations: NA, not applicable; NR, not reported. 
comparison to the other available agents is inherently flawed but can be informative in lieu of direct head-to-head trials. In this case, it suggests that the efficacy of these 3 regimens is similar with PFS of 10-11 months and response rates between $30 \%-40 \%$ (Table 3 ). Further study is needed to determine the best sequencing of the now 7 available regimens. Although everolimus is the only agent that has proven efficacy in a prospective randomized controlled trial after prior TKI failure ${ }^{36}$ sunitinib and sorafenib do appear safe and active after patients have failed prior bevacizumab or the TKIs. ${ }^{37-42}$ For example, in a phase 2 multicenter study, 61 patients who had progressed on bevacizumab received sunitinib $50 \mathrm{mg}$ orally daily on a 4 -week on/2-week off schedule. ${ }^{39}$ The ORR was $23 \%$ with a median duration of response of 44.1 weeks and median PFS of 30.4 weeks. Although direct investigation is needed to inform the most effective sequence, collectively these studies demonstrate that the VEGF inhibitors can have substantial activity sequentially.

In addition to its combination with IFN, several attempts at increasing bevacizumab's efficacy have been evaluated by combining it with other multityrosine kinase and mTOR inhibitors effective in RCC. Multifaceted VEGF blockade both at the level of the ligand with antibodies, such as bevacizumab, and at the receptor with small molecule inhibitors, like sunitinib or sorafenib, would seem to be the most rational and promising. In the small phase 1 studies that have been performed, enhanced antitumor activity has been observed but at the expense of concerning toxicities. ${ }^{43-45}$

Two phase 1 studies have evaluated the combination of sunitinib and bevacizumab. In the dedicated RCC trial, the maximally tolerated dose (MTD) was determined to be sunitinib $50 \mathrm{mg}$ and bevacizumab $10 \mathrm{mg} / \mathrm{kg}$ with an ORR of $52 \%$ in the 25 patients treated. ${ }^{44}$ However, patients experienced significant toxicity with $48 \%$ discontinuing therapy. In the MTD group, grade $\geq 3$ adverse events included hypertension in $83 \%$, proteinuria in $50 \%$, and thrombocytopenia in $50 \%$. A notable pentad of symptoms including hemolytic anemia, thrombocytopenia, proteinuria, hypertension, and renal insufficiency occurred in 8 patients. Two of the patients with severe microangiopathic hemolytic anemia (MAHA) experienced severe hypertension with subsequent development of reversible posterior leukoencephalopathy syndrome (RPLS). Interestingly, with the exception of hypertension, these toxicities were not significantly observed in the phase 1 trial of the same combination in advanced solid tumors. ${ }^{45}$ Of note, only 6 of those 48 patients were RCC patients. Only 1 dose-limiting toxicity of grade 4 hypertension occurred in the dose below the MTD group, and no patient experienced MAHA in this trial. Grade 3 or greater toxicity occurred in $87 \%$ of patients including hypertension $(47 \%)$, fatigue $(24 \%)$, thrombocytopenia $(18 \%)$, proteinuria $(13 \%)$, and hand-foot syndrome (13\%). Although modestly active in this treatment-refractory group, the investigators admit dose modification and delays were frequently necessary for continued treatment particularly with the higher doses of each agent.

Combining bevacizumab with sorafenib, another multiTKI with activity against Raf kinase and VEGFR-2, appears to face similar tolerability challenges. In a phase 1 trial evaluating the combination of sorafenib $200 \mathrm{mg}$ orally twice daily and bevacizumab 5-10 mg/kg every 2 weeks, toxicity would not permit either drug to be escalated to the standard single agent doses. ${ }^{43}$ By a median of 4 cycles, dose reduction of sorafenib was required in $74 \%$ of patients. Although clinical benefit was seen, greater intensity, rapidity, and frequency of toxicity were observed in the form of increased hypertension, massive proteinuria, thrombocytopenia, and hand-foot syndrome.

Bevacizumab's use in combination with mTOR inhibition is also an area of considerable interest. There are at least 4 ongoing trials evaluating the efficacy and safety of bevacizumab and the IV mTOR inhibitor temsirolimus (www.clinical trials.gov, Accessed April 11, 2010). But perhaps the most

Table 3 Efficacy results for the phase 3 VEGF inhibitor randomized controlled trials in treatment-naive advanced RCC

\begin{tabular}{|c|c|c|c|c|c|c|}
\hline Trial & No & PFS, mo & OS, mo & ORR,\% & $\begin{array}{l}\text { Time to } \\
\text { response, mo }\end{array}$ & $\begin{array}{l}\text { Median duration } \\
\text { response, mo }\end{array}$ \\
\hline $\begin{array}{l}\text { Bevacizumab + IFN- } \alpha \text { vs } \\
\text { placebo/IFN- } \alpha \text { (AVOREN) })^{30,31}\end{array}$ & 649 & $\begin{array}{l}\text { I0.2 vs } 5.4 \\
(P=0.000 \mid)\end{array}$ & $\begin{array}{l}23.3 \text { vs } 21.3 \\
\text { (stratified, } P=0.1291)\end{array}$ & 31 vs 13 & 2.2 vs 3.7 & | 3.5 vs $\mid 1.1$ \\
\hline $\begin{array}{l}\text { Bevacizumab + IFN- } \alpha \text { vs } \\
\text { IFN- } \alpha(\text { CALGB 90206) })^{32,33}\end{array}$ & 732 & $\begin{array}{l}8.5 \text { vs } 5.2 \\
(P<0.000 \mathrm{I})\end{array}$ & $\begin{array}{l}18.3 \text { vs } 17.4 \\
\text { (stratified, } P=0.097 \text { ) }\end{array}$ & 25.5 vs I3.I & NR & 11.9 vs 8.7 \\
\hline Sunitinib vs IFN- $\alpha^{62,63}$ & 750 & $\begin{array}{l}\text { I I vs } 5 \\
P<0.00 \text { I }\end{array}$ & $\begin{array}{l}26.4 \text { vs } 21.8 \\
(P=0.05 I, \text { stratified, } \\
P=0.049)\end{array}$ & 47 vs 12 & NR & NR \\
\hline Pazopanib vs BSC/placebo ${ }^{86}$ & 233 & $\begin{array}{l}\text { II.I vs } 2.8 \\
P<0.000 \text { I }\end{array}$ & NR & 32 vs 4 & $3.0^{\mathrm{a}}$ & $14.7^{\mathrm{a}}$ \\
\hline
\end{tabular}

Note: ${ }^{a}$ All treatment groups, not just treatment naïve.

Abbreviations: RCC, renal cell carcinoma; PFS, progression-free survival; OS, overall survival; IFN, interferon; NR, not reported; BSC, best supportive care. 
advanced work has been done with the oral mTOR inhibitor, everolimus. ${ }^{46}$ Two phase 1 trials initially demonstrated the safety and tolerability of bevacizumab and everolimus in combination in advanced solid tumors. Zafar and colleagues ${ }^{47}$ investigated bevacizumab $10 \mathrm{mg} / \mathrm{kg}$ IV every 14 days and everolimus 5 $\mathrm{mg}$ orally daily, escalating to $10 \mathrm{mg}$ daily. No dose-limiting toxicities were observed in the 14 patients enrolled. From this study, the recommended phase 2 dosing of this combination was bevacizumab $10 \mathrm{mg} / \mathrm{kg}$ IV and everolimus $10 \mathrm{mg}$ orally daily. The second phase 1 trial evaluated this combination with the addition of the EGFR-TKI erlotinib in a dose-escalation trial. ${ }^{48}$ The initial dose of bevacizumab $10 \mathrm{mg} / \mathrm{kg}$ IV every 14 days and everolimus $5 \mathrm{mg}$ orally daily was escalated to everolimus $10 \mathrm{mg}$ daily. If the escalated dose of everolimus was tolerable, then erlotinib was added at $75 \mathrm{mg}$ orally daily. No grade 3 toxicities were seen until erlotinib was added. With the addition of erlotinib, 2 of 6 patients experienced grade 3 mucositis and rash. The doses were de-escalated to bevacizumab $5 \mathrm{mg}$ IV, everolimus $5 \mathrm{mg}$ orally daily, and erlotinib $75 \mathrm{mg}$ orally daily with no dose-limiting toxicities.

A phase 2 study of bevacizumab and everolimus has demonstrated efficacy in both treatment-naïve and treatmentrefractory (prior sunitinib and/or sorafenib) patients. ${ }^{46}$ Of the 50 treatment-naïve and 30 pretreated patients, median PFS were 9.1 months and 7.1 months with ORR of $30 \%$ and $23 \%$, respectively. Overall, the regimen was well tolerated with the most common grade 3 or greater toxicities being proteinuria (26\%), mucositis/stomatitis (15\%), fatigue (12\%), and diarrhea $(9 \%)$. The incidence of proteinuria was greater than expected, and 2 patients developed nephrotic syndrome. No symptomatic pneumonitis or MAHA was seen. The authors recognize the hazards of indirectly comparing phase 2 results to phase 3 results of first-line sunitinib or second-line sorafenib, but admit the lack of increase in PFS or response rate compared with the available single agents may not support the combined use. Further study of these agents is needed before firmly drawing conclusions. Our center is accruing a single institution, phase 2 study evaluating this same combination in RCC patients who are refractory to 1 or 2 prior VEGF TKIs. Additionally, a randomized phase 2 study is underway comparing the combination to bevacizumab/IFN in treatment-naïve patients.

In summary, bevacizumab has activity in advanced RCC and holds promise among investigators. As of February 2010, there were at least 50 trials (www.clinicaltrial.gov, Accessed February 10, 2010, "Bevacizumab + Renal Cell") evaluating bevacizumab as monotherapy and in various combination therapies in RCC.

\section{Bevacizumab-related toxicities}

Many of the side effects related to bevacizumab have been discussed in the above sections. Bevacizumab is given rarely as a monotherapy in RCC or other solid tumors, so categorization of its individual side effects has been limited to results of small phase 1 and 2 series and assessment of toxicities deemed unexpected from the other investigational agent. In Yang's small RCC series $(n=76 / 116)$ evaluating bevacizumab monotherapy, the most common toxicities at both the $3 \mathrm{mg} / \mathrm{kg}$ and $10 \mathrm{mg} / \mathrm{kg}$ dosages were hypertension and proteinuria, respectively. ${ }^{15,16}$ The proteinuria was not associated with decreased renal function. In the high-dose arm, epistaxis (8\%), hypertension (14\%; grade 3: $8 \%$ ), hematuria (5\%), and proteinuria (25\%; grade $3: 3 \%)$ were all significantly increased from the placebo.

In the 2 large randomized phase 3 trials comparing its efficacy in combination with IFN, the primary toxicities attributed to bevacizumab included hypertension $(26 \%$; grade $\geq 3: 3 \%-10 \%$ ), proteinuria $(18 \%$; grade $\geq 3: 7 \%-15 \%$ ), and bleeding (33\%; grade $\geq 3: 2 \%-3 \%)$. ${ }^{31,32}$ Well-established IFN-associated toxicities such as fatigue, asthenia, neutropenia, fever, and depression were common in all patients in both trials but increased in the bevacizumab arm. In the AVOREN trial, the IFN-related toxicities occurred with a 10\% higher incidence per patient year in the bevacizumab arm.

Cardiotoxicity with the VEGF inhibitors has warranted further study. Like the multi-TKIs that target VEGF sunitinib and sorafenib, bevacizumab has potential for cardiac toxicity most commonly in the form of hypertension (Table 2). Clinical sequelae of hypertension are rare but can progress to hypertensive crisis, hypertensive encephalopathy, and RPLS. ${ }^{13,49,50}$ RPLS is an underappreciated but recognizable clinicoradiographic syndrome characterized by a reversible cortical dysfunction with symptoms of headache, altered mental function, seizures, vomiting, and visual disturbances including cortical blindness. Characteristic imaging findings include white matter edema most commonly in the bilateral posterior cerebral hemispheres especially the parietaloccipital lobes. ${ }^{51}$ If promptly recognized, it is reversible and controllable with treatment of hypertension, symptom management, and bevacizumab discontinuation.

More recently, the occurrence of bevacizumab-induced hypertension has been postulated to be predictive of response and outcome. This relationship between new or worsening hypertension and improved OS has been reported in solid tumor patients receiving bevacizumab-containing regimens and with other VEGF inhibitors. ${ }^{32,52-57}$ A retrospective analysis of the CALGB trial revealed that $3 \%$ of the IFN-only 
patients developed grade $\geq 2$ hypertension compared with $21 \%$ of the combination arm. ${ }^{32}$ With the exception of a slight increase in the number of patients who had had nephrectomy on the combination arm $(92 \%$ vs $83 \%, P=0.05)$, the authors report that the baseline prognostic factors were balanced between the 2 groups. There was a significant increase in both PFS and OS in patients who experienced grade $\geq 2$ hypertension compared with those who did not at 13.2 months vs 8 months $(P<0.001)$ and 41.6 months and 16.2 months $(P<0.001)$, respectively.

A more concerning toxicity of left ventricular dysfunction has been observed with the multi-TKIs, especially sunitinib. Rates of congestive heart failure and left ventricular dysfunction have been cited as high as $28 \%$ with sunitinib in the RCC and gastrointestinal stromal tumors series. ${ }^{58-66}$ Bevacizumab's reported rate of congestive heart failure is significantly less at $<1 \%$ in the phase $3 \mathrm{RCC}$ trials and $1.2 \%-3.8 \%$ in the solid tumor trials. ${ }^{13,31-33}$ Previous exposure to anthracyclines, prior irradiation to the chest wall, and baseline left ventricular systolic dysfunction may increase risk. Although many mechanistic theories implicate inhibition of PDGFR, ribosomal S6 kinase, and RAF1 kinase and their resultant downstream effects, the exact mechanisms behind this cardiotoxicity remain unclear. ${ }^{67}$ Other etiologies behind the treatment-induced hypertension include vascular rarefaction, endothelial dysfunction, and/or altered nitrous oxide metabolism rather than a direct result of VEGF inhibition or effects on the renovascular or adrenergic system. ${ }^{68}$ Bevacizumab's lower incidence of left ventricular systolic dysfunction, but similarly high rates of hypertension, lend support to the cardiotoxicity being a non-VEGF and nonhypertensionmediated event.

The incidence of arterial thromboembolic events has been notable with bevacizumab. In a pooled analysis of the randomized clinical trials of patients receiving bevacizumab in combination with chemotherapy $(n=1745), 4.4 \%$ compared with $1.9 \%$ being treated with chemotherapy alone experienced arterial thromboembolic events (cerebral infarction, transient ischemic attacks, MI, angina, and a variety of other aterial thromboembolic events). ${ }^{13}$ In the advanced RCC trials, there was an increased incidence of cardiac ischemia/infarction ( $1 \%$ vs $0 \%)$ and thrombosis/ embolism ( $4 \%$ vs $<1 \%-2 \%)$ in the bevacizumab-treated patients. $^{30,32}$

Proteinuria is a well-characterized complication of bevacizumab. In the RCC randomized controlled trials, the incidence of all grades was $18 \%-71 \%$ with bevacizumab therapy compared with $3 \%-10 \%$ in the IFN monotherapy group. VEGF is constitutively expressed by podocytes, and normal glomerular capillary endothelial cells have VEGF receptors. ${ }^{69}$ Using murine models, Sugimoto and colleagues ${ }^{70}$ demonstrated that anti-VEGF neutralizing antibodies and soluble VEGFR-1 protein (sFlt-1) induce proteinuria by rapid glomerular endothelial cell detachment and hypertrophy, which was associated with a concomitant downregulation of nephrin, a protein integral to the glomerular filtration process. Further investigating how bevacizumab might directly induce renal thrombotic microangiopathy, Eremina and colleagues ${ }^{69}$ developed a conditional gene murine model that targeted only podocytes, which are the major source of glomerular VEGF production. They confirmed that VEGF production by podocytes is necessary for glomerular endothelium integrity and that disruption by drug or genetic manipulation results in renal pathology consistent with the thrombotic microangiopathy induced by bevacizumab. It is unclear whether combinations of agents or sequential use of TKIs may exacerbate proteinuria and renal toxicity. There has been little reported proteinuria with everolimus monotherapy, but a higher than expected incidence of proteinuria was seen in the phase 2 combination trial with bevacizumab. ${ }^{46}$ Our anecdotal experience in an ongoing phase 2 trial evaluating bevacizumab and everolimus is consistent with the published phase 2 study as well. We recommend checking a urine protein before each dose of bevacizumab and following the hemoglobin, platelets, and creatinine for signs of MAHA and renal toxicity.

The risk of hemorrhage with bevacizumab is a black box warning and the FDA notes that severe or fatal hemorrhage, including hemoptysis, gastrointestinal bleeding, central nervous system hemorrhage, epistaxis, and vaginal bleeding, has occurred up to 5-fold more in patients receiving bevacizumab. ${ }^{13}$ In the 2 RCC trials, the incidence of all grade hemorrhage/bleeding was 5\%-33\% with bevacizumab compared with $<1 \%-9 \%$ in the control group; $1 \%-3 \%$ of the bevacizumab-attributable bleeding was grade $\geq 3$ in nature.

Wound-healing complications such as wound dehiscence and delayed healing have been reported in patients receiving bevacizumab. Animal models demonstrate that antibody neutralization of VEGF inhibits wound granulation tissue and that VEGF stimulates epithelialization and collagen deposition in wounds. ${ }^{71,72}$ The incidence of wound-healing complications in the phase $3 \mathrm{RCC}$ trials was only $1 \%$ and not significantly increased compared with the control group. ${ }^{31}$ This complication may be more germane to the growing use 
of these agents preoperatively to achieve downsizing of the tumor and safer surgeries. ${ }^{73-78}$ M.D. Anderson has reported the largest series of patients undergoing VEGF inhibitor therapy before cytoreductive open or laparoscopic nephrectomy. ${ }^{76}$ They retrospectively analyzed 44 patients who received either bevacizumab, sorafenib, or sunitinib before nephrectomy and compared the complications to a matched cohort of 58 patients who received up-front surgery. Bevacizumab was held for at least 4 weeks before surgery. Their initial review reported no difference in operative time, estimated blood loss, amount of transfused blood products, or overall hospital stay, but an updated analysis 2 years later demonstrated significant differences in superficial wound-healing complications (odds ratio $=19.7, P<0.01)$ and delays in starting adjuvant therapy by about 17 days (48.8 days vs 31.7 days, $P<0.01) .{ }^{79}$

A prospective study evaluated the presurgical feasibility and safety of bevacizumab (with or without erlotinib) in 50 treatment-naïve metastatic RCC patients. ${ }^{75}$ After 2 cycles of presurgical bevacizumab, $52 \%$ experienced primary tumor reduction and the majority (84\%) underwent nephrectomy. There was a notable incidence of wound dehiscence and delayed wound healing at 4 weeks postnephrectomy. Wound dehiscence complicated the postoperative course of 5 patients requiring either treatment discontinuation $(n=3)$ or delay ( $\mathrm{n}=2$, median time delay: 20.5 days). The incidence of delayed superficial wound healing was significantly higher (21\% vs $2 \%, P<0.001)$ with presurgical bevacizumab than in a historic matched cohort of 101 patients who had undergone up-front surgery. Given the estimated half-life of approximately 20 days, bevacizumab should be held for at least 4-6 weeks before surgery when possible and should be discontinued in patients with severe wound-healing complications. ${ }^{13}$

In summary, bevacizumab commonly induces hypertension and proteinuria and in combination with IFN, fatigue and asthenia. Concerning complications can include thromboembolic events, hemorrhage, gastrointestinal perforation, and rare infusion-related reactions. Its use may offer advantages compared with its first-line tyrosine kinase competitors in terms of no or lower incidences of hand-foot syndrome, left ventricular systolic dysfunction, anemia, thrombocytopenia, thyroid dysfunction, and liver toxicity (Table 2).

\section{Conclusions}

Bevacizumab plus IFN is now an FDA-approved option for first-line therapy in metastatic RCC. Acknowledging the limitations of indirect comparison, the regimen likely has equivalent efficacy to the other first-line, FDA-approved, VEGF-targeted agents, sunitinib and pazopanib. Enthusiasm for its use in combination with IFN is blunted by the increased costs and potential infusion-related reactions associated with its IV administration, the IFN-related toxicities, and the possible patient dissatisfaction with the inconvenience of its nonoral formulation. Advantages include a somewhat different toxicity profile from the multi-TKIs and assurance of patient compliance with its IV administration. Ongoing studies are assessing its utility in combination with other targeted agents and immunotherapies and will revisit its use as a monotherapy. Identification of patient and tumor-specific biomarkers to inform our choice of first-line therapy as well as the sequential use of the available agents to treat RCC is imperative.

\section{Disclosure}

Dr Harshman and Dr Srinivas receive investigational drug and research funding from Genentech, Inc., Novartis Pharmaceuticals, and GlaxoSmithKline.

\section{References}

1. Yagoda A, Petrylak D, Thompson S. Cytotoxic chemotherapy for advanced renal cell carcinoma. Urol Clin North Am. 1993;20: 303-321.

2. Lonser RR, Glenn GM, Walther M, et al. von Hippel-Lindau disease. Lancet. 2003;361:2059-2067.

3. Gallou C, Joly D, Mejean A, et al. Mutations of the VHL gene in sporadic renal cell carcinoma: definition of a risk factor for VHL patients to develop an RCC. Hum Mutat. 1999;13:464-475.

4. Gnarra JR, Tory K, Weng Y, et al. Mutations of the VHL tumour suppressor gene in renal carcinoma. Nat Genet. 1994;7:85-90.

5. Kim W, Kaelin WG Jr. The von Hippel-Lindau tumor suppressor protein: new insights into oxygen sensing and cancer. Curr Opin Genet Dev. 2003;13:55-60.

6. Kondo K, Yao M, Yoshida M, et al. Comprehensive mutational analysis of the VHL gene in sporadic renal cell carcinoma: relationship to clinicopathological parameters. Genes Chromosomes Cancer. 2002; 34:58-68.

7. Sukosd F, Kuroda N, Beothe T, Kaur AP, Kovacs G. Deletion of chromosome 3p14.2-p25 involving the VHL and FHIT genes in conventional renal cell carcinoma. Cancer Res. 2003;63:455-457.

8. Latif F, Tory K, Gnarra J, et al. Identification of the von Hippel-Lindau disease tumor suppressor gene. Science. 1993;260:1317-1320.

9. Maxwell PH, Wiesener MS, Chang GW, et al. The tumour suppressor protein VHL targets hypoxia-inducible factors for oxygen-dependent proteolysis. Nature. 1999;399:271-275.

10. Ferrara N, Gerber HP, LeCouter J. The biology of VEGF and its receptors. Nat Med. 2003;9:669-676.

11. Leung DW, Cachianes G, Kuang WJ, Goeddel DV, Ferrara N. Vascular endothelial growth factor is a secreted angiogenic mitogen. Science. 1989;246:1306-1309.

12. Presta LG, Chen H, O'Connor SJ, et al. Humanization of an anti-vascular endothelial growth factor monoclonal antibody for the therapy of solid tumors and other disorders. Cancer Res. 1997;57:4593-4599.

13. Bevacizumab [package insert]. Genentech; 2009. South San Francisco, CA, USA. 
14. Gordon MS, Margolin K, Talpaz M, et al. Phase I safety and pharmacokinetic study of recombinant human anti-vascular endothelial growth factor in patients with advanced cancer. J Clin Oncol. 2001;19: 843-850.

15. Yang JC. Bevacizumab for patients with metastatic renal cancer: an update. Clin Cancer Res. 2004;10:6367S-6370S.

16. Yang JC, Haworth L, Sherry RM, et al. A randomized trial of bevacizumab, an anti-vascular endothelial growth factor antibody, for metastatic renal cancer. $N$ Engl J Med. 2003;349:427-434.

17. Elaraj DM, White DE, Steinberg SM, et al. A pilot study of antiangiogenic therapy with bevacizumab and thalidomide in patients with metastatic renal cell carcinoma. J Immunother. 2004;27:259-264.

18. D'Amato RJ, Loughnan MS, Flynn E, Folkman J. Thalidomide is an inhibitor of angiogenesis. Proc Natl Acad Sci U S A. 1994;91: 4082-4085.

19. Kenyon BM, Browne F, D'Amato RJ. Effects of thalidomide and related metabolites in a mouse corneal model of neovascularization. Exp Eye Res. 1997;64:971-978.

20. Eisen T, Boshoff C, Mak I, et al. Continuous low dose thalidomide: a phase II study in advanced melanoma, renal cell, ovarian and breast cancer. Br J Cancer. 2000;82:812-817.

21. Escudier B, Lassau N, Couanet D, et al. Phase II trial of thalidomide in renal-cell carcinoma. Ann Oncol. 2002;13:1029-1035.

22. Minor DR, Monroe D, Damico LA, et al. A phase II study of thalidomide in advanced metastatic renal cell carcinoma. Invest New Drugs. 2002; 20:389-393

23. Motzer RJ, Berg W, Ginsberg M, et al. Phase II trial of thalidomide for patients with advanced renal cell carcinoma. J Clin Oncol. 2002; 20:302-306.

24. Hainsworth JD, Sosman JA, Spigel DR, et al. Treatment of metastatic renal cell carcinoma with a combination of bevacizumab and erlotinib. J Clin Oncol. 2005;23:7889-7896.

25. Ciardiello F, Caputo R, Bianco R, et al. Inhibition of growth factor production and angiogenesis in human cancer cells by ZD1839 (Iressa), a selective epidermal growth factor receptor tyrosine kinase inhibitor. Clin Cancer Res. 2001;7:1459-1465.

26. Riedel F, Gotte K, Li M, Hormann K, Grandis JR. EGFR antisense treatment of human HNSCC cell lines down-regulates VEGF expression and endothelial cell migration. Int J Oncol. 2002;21:11-16.

27. Bukowski RM, Kabbinavar FF, Figlin RA, et al. Randomized phase II study of erlotinib combined with bevacizumab compared with bevacizumab alone in metastatic renal cell cancer. J Clin Oncol. 2007;25:4536-4541.

28. McDermott DF, Regan MM, Clark JI, et al. Randomized phase III trial of high-dose interleukin-2 versus subcutaneous interleukin-2 and interferon in patients with metastatic renal cell carcinoma. J Clin Oncol. $2005 ; 23: 133-141$

29. Motzer RJ, Bacik J, Murphy BA, Russo P, Mazumdar M. Interferonalfa as a comparative treatment for clinical trials of new therapies against advanced renal cell carcinoma. J Clin Oncol. 2002;20: 289-296.

30. Escudier B, Bellmunt J, Negrier S, et al. Phase III trial of bevacizumab plus interferon alfa-2a in patients with metastatic renal cell carcinoma (AVOREN): final analysis of overall survival. J Clin Oncol. 2010:12144-12150.

31. Escudier B, Pluzanska A, Koralewski P, et al. Bevacizumab plus interferon alfa-2a for treatment of metastatic renal cell carcinoma: a randomised, double-blind phase III trial. Lancet. 2007;370: 2103-2111.

32. Rini BI, Halabi S, Rosenberg JE, et al. Phase III trial of bevacizumab plus interferon alfa versus interferon alfa monotherapy in patients with metastatic renal cell carcinoma: final results of CALGB 90206. J Clin Oncol. 2010:2137-2143.

33. Rini BI, Halabi S, Rosenberg JE, et al. Bevacizumab plus interferon alfa compared with interferon alfa monotherapy in patients with metastatic renal cell carcinoma: CALGB 90206. J Clin Oncol. 2008;26: $5422-5428$.
34. Rini BI, Halabi S, Taylor J, Small EJ, Schilsky RL; for Cancer and Leukemia Group B 90206. A randomized phase III trial of interferon-alpha or interferon-alpha plus anti-vascular endothelial growth factor antibody (bevacizumab) in metastatic renal cell carcinoma. Clin Cancer Res. 2004;10:2584-2586.

35. Melichar B, Koralewski P, Ravaud A, et al. First-line bevacizumab combined with reduced dose interferon-alpha2a is active in patients with metastatic renal cell carcinoma. Ann Oncol. 2008;19: 1470-1476.

36. Motzer RJ, Escudier B, Oudard S, et al. Efficacy of everolimus in advanced renal cell carcinoma: a double-blind, randomised, placebo-controlled phase III trial. Lancet. 2008;372:449-456.

37. Dudek AZ, Zolnierek J, Dham A, Lindgren BR, Szczylik C. Sequential therapy with sorafenib and sunitinib in renal cell carcinoma. Cancer. 2009;115:61-67.

38. Eichelberg C, Heuer R, Chun FK, et al. Sequential use of the tyrosine kinase inhibitors sorafenib and sunitinib in metastatic renal cell carcinoma: a retrospective outcome analysis. Eur Urol. 2008;54: 1373-1378.

39. Rini BI, Michaelson MD, Rosenberg JE, et al. Antitumor activity and biomarker analysis of sunitinib in patients with bevacizumabrefractory metastatic renal cell carcinoma. J Clin Oncol. 2008;26: 3743-3748.

40. Stadler WM, Figlin RA, McDermott DF, et al. Safety and efficacy results of the advanced renal cell carcinoma sorafenib expanded access program in North America. Cancer. 1272;116:1272-1280.

41. Tamaskar I, Garcia JA, Elson P, et al. Antitumor effects of sunitinib or sorafenib in patients with metastatic renal cell carcinoma who received prior antiangiogenic therapy [discussion 6]. J Urol. 2008; 179:81-86

42. Zimmermann K, Schmittel A, Steiner U, et al. Sunitinib treatment for patients with advanced clear-cell renal-cell carcinoma after progression on sorafenib. Oncology. 2009;76:350-354.

43. Azad NS, Posadas EM, Kwitkowski VE, et al. Combination targeted therapy with sorafenib and bevacizumab results in enhanced toxicity and antitumor activity. J Clin Oncol. 2008;26:3709-3714.

44. Feldman DR, Baum MS, Ginsberg MS, et al. Phase I trial of bevacizumab plus escalated doses of sunitinib in patients with metastatic renal cell carcinoma. J Clin Oncol. 2009;27:1432-1439.

45. Rini BI, Garcia JA, Cooney MM, et al. A phase I study of sunitinib plus bevacizumab in advanced solid tumors. Clin Cancer Res. 2009;15: $6277-6283$

46. Hainsworth JD, Spigel DR, Burris HA III, et al. Phase II trial of bevacizumab and everolimus in patients with advanced renal cell carcinoma. J Clin Oncol. 2010:2131-2136.

47. Zafar Y, Bendell J, Lager J, et al. Preliminary results of a phase I study of bevacizumab (BV) in combination with everolimus (E) in patients with advanced solid tumors. In: American Society of Clinical Oncology Annual Meeting; June 2006; Atlanta, GA.

48. Bendell JC, George D, Nixon A, et al. Results of a phase I study of bevacizumab (BV), everolimus (EV), and erlotinib (E) in patients with advanced solid tumors. In: American Society of Clinical Oncology Annual Meeting; June 2007; Chicago, IL.

49. Glusker P, Recht L, Lane B. Reversible posterior leukoencephalopathy syndrome and bevacizumab [discussion 2]. $N$ Engl J Med. 2006; 354:980-981.

50. Ozcan C, Wong SJ, Hari P. Reversible posterior leukoencephalopathy syndrome and bevacizumab [discussion 2]. N Engl J Med. 2006; 354:980-982.

51. Hinchey J, Chaves C, Appignani B, et al. A reversible posterior leukoencephalopathy syndrome. $N$ Engl J Med. 1996;334:494-500.

52. Bibb JL, Lee S, Lu D, Lee F. Increase in systolic (SBP) and decrease in diastolic blood pressure (DBP) may predict clinical benefit $(\mathrm{CB})$ of bevacizumab (B) in patients (PTS) with metastatic colorectal cancer $(\mathrm{mCRC})$ treated with cytotoxic agents $(\mathrm{CA})$ [abstract 450]. ASCO Gastrointestinal Cancers Symposium; January 2007; Orlands, FL, USA. 
53. Dahlberg SE, Sandler AB, Brahmer JR, Schiller JH, Johnson DH. Clinical course of advanced non-small-cell lung cancer patients experiencing hypertension during treatment with bevacizumab in combination with carboplatin and paclitaxel on ECOG 4599. J Clin Oncol. 2010;28:949-954.

54. Friberg GKK, Vokes EE, Kindler HL. Early hypertension (HTN) as a potential pharmacodynamic (PD) marker for survival in pancreatic cancer (PC) patients (pts) treated with bevacizumab (B) and gemcitabine (G) [abstract 3020]. Proceedings of Am Soc Clin Oncol. 2005. www.asco.org.

55. Rini B, Schiller J, Fruehauf JP, et al. Association of diastolic blood pressure $(\mathrm{dBP})>90 \mathrm{mmHg}$ with overall survival (OS) in patients treated with axitinib (AG- 013736) [abstract 3543]. J Clin Oncol. 2008;26. www.asco.org.

56. Scartozzi M, Galizia E, Chiorrini S, et al. Arterial hypertension correlates with clinical outcome in colorectal cancer patients treated with first-line bevacizumab. Ann Oncol. 2009;20:227-230.

57. Schneider BP, Wang M, Radovich M, et al. Association of vascular endothelial growth factor and vascular endothelial growth factor receptor-2 genetic polymorphisms with outcome in a trial of paclitaxel compared with paclitaxel plus bevacizumab in advanced breast cancer: ECOG 2100. J Clin Oncol. 2008;26:4672-4678.

58. Choueiri TK, Schutz FA, Je Y, Rosenberg JE, Bellmunt J. Risk of arterial thromboembolic events with sunitinib and sorafenib: a systematic review and meta-analysis of clinical trials. J Clin Oncol. 2010;28:2280-2285.

59. Chu TF, Rupnick MA, Kerkela R, et al. Cardiotoxicity associated with tyrosine kinase inhibitor sunitinib. Lancet. 2007;370:2011-2019.

60. Khakoo AY, Kassiotis CM, Tannir N, et al. Heart failure associated with sunitinib malate: a multitargeted receptor tyrosine kinase inhibitor. Cancer. 2008;112:2500-2508.

61. Lenihan DJ. Tyrosine kinase inhibitors: can promising new therapy associated with cardiac toxicity strengthen the concept of teamwork? J Clin Oncol. 2008;26:5154-5155.

62. Motzer RJ, Hutson TE, Tomczak P, et al. Overall survival and updated results for sunitinib compared with interferon alfa in patients with metastatic renal cell carcinoma. J Clin Oncol. 2009;27:3584-3590.

63. Motzer RJ, Hutson TE, Tomczak P, et al. Sunitinib versus interferon alfa in metastatic renal-cell carcinoma. $N$ Engl J Med. 2007;356:115-124.

64. Sunitinib maleate [package insert]. Pfizer; 2010

65. Schmidinger M, Zielinski CC, Vogl UM, et al. Cardiac toxicity of sunitinib and sorafenib in patients with metastatic renal cell carcinoma. J Clin Oncol. 2008;26:5204-5212.

66. Telli ML, Witteles RM, Fisher GA, Srinivas S. Cardiotoxicity associated with the cancer therapeutic agent sunitinib malate. Ann Oncol. 2008; 19:1613-1618.

67. Force T, Krause DS, Van Etten RA. Molecular mechanisms of cardiotoxicity of tyrosine kinase inhibition. Nat Rev Cancer. 2007;7: 332-344.

68. Veronese ML, Mosenkis A, Flaherty KT, et al. Mechanisms of hypertension associated with BAY 43-9006. J Clin Oncol. 2006; 24:1363-1369.

69. Eremina V, Jefferson JA, Kowalewska J, et al. VEGF inhibition and renal thrombotic microangiopathy. N Engl J Med. 2008;358:1129-1136.
70. Sugimoto H, Hamano Y, Charytan D, et al. Neutralization of circulating vascular endothelial growth factor (VEGF) by anti-VEGF antibodies and soluble VEGF receptor 1 (sFlt-1) induces proteinuria. J Biol Chem. 2003;278:12605-12608.

71. Bao P, Kodra A, Tomic-Canic M, et al. The role of vascular endothelial growth factor in wound healing. J Surg Res. 2009;153:347-358.

72. Howdieshell TR, Callaway D, Webb WL, et al. Antibody neutralization of vascular endothelial growth factor inhibits wound granulation tissue formation. J Surg Res. 2001;96:173-182.

73. Amin C, Wallen E, Pruthi RS, et al. Preoperative tyrosine kinase inhibition as an adjunct to debulking nephrectomy. Urology. 2008; 72:864-868.

74. Cowey CL, Amin C, Pruthi RS, et al. Neoadjuvant clinical trial with sorafenib for patients with stage II or higher renal cell carcinoma J Clin Oncol. 1502;28:1502-1507.

75. Jonasch E, Wood CG, Matin SF, et al. Phase II presurgical feasibility study of bevacizumab in untreated patients with metastatic renal cell carcinoma. J Clin Oncol. 2009;27:27.

76. Margulis V, Matin SF, Tannir N, et al. Surgical morbidity associated with administration of targeted molecular therapies before cytoreductive nephrectomy or resection of locally recurrent renal cell carcinoma. J Urol. 2008;180:94-98.

77. Thomas AA, Rini BI, Stephenson AJ, et al. Surgical resection of renal cell carcinoma after targeted therapy. J Urol. 2009;16:16.

78. Wood CG, Margulis V. Neoadjuvant (presurgical) therapy for renal cell carcinoma: a new treatment paradigm for locally advanced and metastatic disease. Cancer. 2009;115:2355-2360.

79. Rini BI. Tyrosine-kinase inhibitors in a presurgical setting. Presentation at: 2009 American Society of Clinical Oncology's Genitourinary Cancers Symposium; March 2009; Ordando, FL, USA.

80. Sorafenib [package insert]. Bayer Pharmaceutical; 2009. Wayne, NJ, USA.

81. Escudier B, Eisen T, Stadler WM, et al. Sorafenib in advanced clear-cell renal-cell carcinoma. $N$ Engl J Med. 2007;356:125-134.

82. Escudier B, Eisen T, Stadler WM, et al. Sorafenib for treatment of renal cell carcinoma: final efficacy and safety results of the phase III treatment approaches in renal cancer global evaluation trial. $J$ Clin Oncol. 2009;27:3312-3318.

83. Escudier B, Szczylik C, Hutson TE, et al. Randomized phase II trial of first-line treatment with sorafenib versus interferon alfa-2a in patients with metastatic renal cell carcinoma. J Clin Oncol. 2009; $27: 1280-1289$

84. Votrient [prescribing information]. GlaxoSmithKline; 2010. Research Triangle, NC, USA.

85. Hutson TE, Davis ID, Machiels JP, et al. Efficacy and safety of pazopanib in patients with metastatic renal cell carcinoma. J Clin Oncol. 2009;28:475-480.

86. Sternberg CN, Davis ID, Mardiak J, et al. Pazopanib in locally advanced or metastatic renal cell carcinoma: results of a randomized phase III trial. J Clin Oncol. 2010;28:1061-1068.
OncoTargets and Therapy

\section{Publish your work in this journal}

OncoTargets and Therapy is an international, peer-reviewed, open access journal focusing on the pathological basis of all cancers, potential targets for therapy and treatment protocols employed to improve the management of cancer patients. The journal also focuses on the impact of management programs and new therapeutic agents and protocols on

\section{Dovepress}

patient perspectives such as quality of life, adherence and satisfaction. The manuscript management system is completely online and includes a very quick and fair peer-review system, which is all easy to use. Visit http://www.dovepress.com/testimonials.php to read real quotes from published authors. 